\title{
Infection and colonization by Stenotrophomonas maltophilia: antimicrobial susceptibility and clinical background of strains isolated at a tertiary care centre in Hungary
}

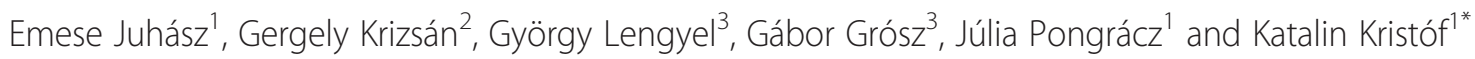

\begin{abstract}
Background: Stenotrophomonas maltophilia is an important opportunistic, mainly nosocomial pathogen that emerged in the last decades worldwide. Due to its inherent extended antibiotic resistance, therapeutic options are strongly limited. New resistance mechanisms in S. maltophilia make antibiotic therapy even more difficult. The aim of our study was to investigate the antimicrobial resistance of S. maltophilia isolates collected in our laboratory and to reveal related clinical background.
\end{abstract}

Method: Consecutive non-duplicate S. maltophilia isolates $(n=160)$ were collected in a three-year period. Conventional methods, automated identification system and MALDI-TOF MS was used for identification, ERIC-PCR for genetic relationship analysis and broth microdilution method to determine the susceptibility for trimethoprim/ sulfamethoxazole (SXT), ciprofloxacin, levofloxacin, moxifloxacin, colistin, doxycycline and tigecycline. Clinical final reports were used retrospectively to collect clinical information.

Results: ERIC-PCR revealed large heterogeneity. Trimethoprim/sulfamethoxazole, moxifloxacin and levofloxacin were found to be the most effective agents with MIC50/MIC90 0.5/1, 0.25/1, 1/2 mg/l, respectively. Seventy percent of patients with S. maltophilia infection were treated in intensive care units. All-cause mortality rate was $45 \%$. Nearly $70 \%$ of the isolates were collected from polymicrobial infections/colonizations.

Conclusions: Trimethoprim/sulfamethoxazole is the most potent antibiotic agent against S. maltophilia. In case of SXT hypersensitivity, intolerance or resistance, fluoroquinolones are alternative therapeutic options. Missing clinical breakpoints, consensus antibiotic susceptibility testing guidelines and clinical trials make the interpretation of antibiotic susceptibility testing results difficult. The indirect pathogenicity of S. maltophilia in polymicrobial infections or colonizations has to be taken into consideration.

Keywords: Stenotrophomonas maltophilia, Antibiotic susceptibility

\section{Background}

Stenotrophomonas maltophilia has emerged as an important opportunistic and nosocomial pathogen in recent years worldwide $[1,2]$. Behind Pseudomonas aeruginosa and Acinetobacter baumannii, S. maltophilia is the third most common non-fermenting Gram-negative bacillus responsible for healthcare-associated infections [1]. Community-

\footnotetext{
* Correspondence: kristof.katalin@med.semmelweis-univ.hu 'Diagnostic Laboratory of Clinical Microbiology, Institute of Laboratory Medicine, Semmelweis University, Budapest, Hungary

Full list of author information is available at the end of the article
}

acquired S. maltophilia infections have also been reported [3]. Pneumonia and bacteraemia are the most frequent infections [1,2]. Due to its inherent extended antibiotic resistance, therapeutic options are strongly limited $[1,2,4]$. Currently only trimethoprim/sulfamethoxazole (SXT) is recommended for therapy, but some circumstances (hypersensitivity of the patient, resistance of the bacterium) can limit the use of this drug [1,2]. In such cases, susceptibility of S. maltophilia isolates for other antimicrobials must be tested, even if clinical evidences for their efficacy are lacking yet [4]. Increasing number of 
patients at risk (immunocompromised patients, cancer patients, patients undergoing long-term intensive care, etc.), the necessity of the usage of broad-spectrum antibiotics such as carbapenems (which cause selective pressure for the inherently carbapenem-resistant S. maltophilia) and the natural features of this bacterium (biofilm forming ability, colonizing moist hospital environments) together mean that $S$. maltophilia is a continuous threat we have to face $[1,2,5]$. The high attributable mortality rates and poor outcomes reported in $S$. maltophilia infection makes the spread of this bacterium even more worrisome [6]. The emergence of new resistance mechanisms in S. maltophilia requires substantial monitoring and reporting of antibiotic suspectibility of clinical isolates [5].

The aim of our study was to investigate the antimicrobial resistance of $S$. maltophilia isolates collected in Diagnostic Laboratory of Clinical Microbiology, Institute of Laboratory Medicine, Semmelweis University (Budapest, Hungary) and to reveal whether isolates were infective or colonizers, the type of infections, the predisposing factors of infected patients, antibiotic therapy and the outcome of infections.

\section{Materials and methods}

A total of 160 consecutive non-duplicate S. maltophilia isolates from a three-year collection period (2009-2011) were investigated in our study. The identification of the isolates was performed with conventional methods, VITEK 2 Gram-negative identification cards (bioMérieux, Marcy l'Etoile) and additionally by MALDI-TOF mass spectrometry (Bruker Daltonics, Bremen). For MALDITOF MS identification, the direct smear and $1 \mu \mathrm{L}$ alphaCyano-4-hydroxycinnamic acid matrix overlay method was applied [7]. Parameters of the device were set according to the recommendation of Bruker. Identifications were assigned using the Bruker Biotyper 2.0 software. Biotyper score $\geq 2.0$ were accepted as valid species level identification. Identification results as "Stenotrophomonas maltophilia", "Stenotrophomonas maltophilia (Pseudomonas beteli)", "Stenotrophomonas maltophilia (Pseudomonas hibiscicola)" were all accepted. Pseudomonas hibiscicola and P. beteli are belonging to Stenotrophomonas rRNA lineage [8].

The isolates were divided into two groups: isolates of patients who were infected by $S$. maltophilia and of those who were colonized only. Infection or colonization was distinguished according to clinical diagnoses given in final reports. Selected cases were discussed with physician to reveal clinical relevance of isolates. The definitive diagnosis of infection was clinically established. Colonization was defined as the presence of S. maltophilia on skin, mucous membranes, in wounds, or in excretions or secretions without causing adverse clinical signs or symptoms. Isolates of infected patients $(n=100)$ were cultured from blood $(\mathrm{n}=25)$, bronchoalveolar lavage sample $(\mathrm{n}=30)$, tracheal aspirate $(\mathrm{n}=31)$, sputum $(\mathrm{n}=7)$, central venous catheter $(n=4)$, peritoneal fluid $(n=3)$. Isolates of colonized patients $(n=60)$ were cultured from rectal swab $(n=11)$, urine $(n=8)$, ear swab $(n=6)$, throat - $(n=$ $3)$, nose - $(n=4)$, eye swab $(n=7)$, catheter $(n=1)$, sputum $(n=7)$, tracheal aspirate $(n=7)$ and wound sample $(n=6)$.

The minimal inhibitory concentrations (MICs) of seven antibiotics were determined by the reference broth microdilution method in cation-adjusted Mueller-Hinton broth [9]. MIC values of the invasive isolates were tested by agar dilution method and by gradient diffusion test, too [9-11]. The antibiotics tested included SXT (0.25-128 mg/L), ciprofloxacin $(0.5-256 \mathrm{mg} / \mathrm{L})$, moxifloxacin $(0.064-32 \mathrm{mg} / \mathrm{L})$, levofloxacin (0.064-32 $\mathrm{mg} / \mathrm{L})$, colistin $(0.25-256 \mathrm{mg} / \mathrm{L})$, doxycycline (0.064-32 $\mathrm{mg} / \mathrm{L})$ and tigecycline (0.064$32 \mathrm{mg} / \mathrm{L})$. The European Committee on Antimicrobial Susceptibility Testing (EUCAST) has $S$. maltophilia specific clinical breakpoint only for SXT [12]. Therefore, non-species related breakpoints of EUCAST were applied for fluoroquinolons and tigecycline. For doxycyline - due to absence of non-species related breakpoints - the epidemiological cut-off (ECOFF) value of S. maltophilia $(8 \mathrm{mg} / \mathrm{L})$ was applied. For colistin - lacking non-species related break-points and approved ECOFF - Pseudomonas sp. specific breakpoint (4 mg/L) was used. Escherichia coli ATCC 25922, Pseudomonas aeruginosa ATCC 27853 and Staphylococcus aureus ATCC 29213 were used as quality control strains.

Enterobacterial Repetitive Intergenic Consensus PCR (ERIC-PCR) was used for molecular typing of isolates, as described by Silbert et al. [13]. Isolates from the same ward were tested within the same PCR amplification and gel electrophoreses run. Band patterns obtained by ERIC-PCR were visually evaluated in the absence of appropriate software. Isolates with two or more different bands were interpreted as unrelated.

Clinical data and laboratory findings (white blood cell number (WBC), C-reactive protein (CRP) and procalcitonin $(\mathrm{PCT})$ ) of patients at the time of $S$. maltophilia infections were collected retrospectively from clinical final reports. Details of antibiotic therapy used in S. maltophilia infections were investigated.

Patient characteristics were tested for their association with overall mortality. First, univariate analysis using chi-squere test or Fisher exact test was performed. A pvalue of $<0.05$ was considered as significant. Variables with significant association with mortality in the univariate analysis were entered in a multivariate forward stepwise logistic regression model to identify independent risk factors for death. The odds ratio (OR) with the corresponding 95\% confidence interval (95\% CI) for each variables were calculated. A p-value of $<0.05$ was considered indicative of statistical significance. Stata 12 
software (StataCorp LP, USA) was used for the statistical analysis.

\section{Results}

Biotyper 2.0 softver of Bruker MALDI-TOF MS classified $77 \%$ of the isolates as S. maltophilia, $16 \%$ as S. maltophilia (P. hibiscicola) and 7\% as S. maltophilia (P. beteli).

ERIC-PCR resulted in highly diverse patterns, however seven identical patterns among colonizer (4 times 2 isolates and 3 times 3 isolates with the same band patterns) and twelve among infective isolates (6 times 2 isolates, 4 times 3 isolates and once 5 isolates and once 6 isolates with the same band patterns) were found. In order to prevent the distortion of values by clonal isolates, 10 colonizer and 23 infective isolates were excluded from the final evaluation of antibiotic susceptibility testing results.

The results obtained with microdilution, gradient diffusion and agar dilution methods were in concordance. However, results obtained with agar dilution - especially in case of SXT - were difficult to evaluate due to slight growth of microcolonies at adjacent antibiotic concentrations. The MIC50 and MIC90 values, MIC ranges and susceptibility in \% of selected isolates with different ERIC-PCR patterns were summarized in Table 1. However, MIC values did not differ significantly if the total of 100 infective and 60 colonizer isolates were evaluated.

The susceptibility rates of infective and colonizer S. maltophilia isolates were compared. Both groups showed high rate of susceptibility to SXT. Apart from ciprofloxacin, the infective isolates had higher rates of non-susceptibility than had colonizers. Non-susceptibility rate for colistin and tigecycline were 1.2-1.3 times higher in the infective group.

The analysis of clinical background of 100 infective isolates revealed that $70 \%$ were cultured from patients admitted to intensive care units (ICU). Sixty-two isolates were obtained from patients with pneumonia (12 of them developing respiratory failure). Forty-six patients had sepsis (23 of them developing severe sepsis, septic shock or multiorgan failure), in 19 cases S. maltophilia was considered as the ethiological agent. The comorbidity was chronic obstructive pulmonary disease (COPD) in 19 cases and malignancy in 19 cases, respectively. Nine patients were immunsuppressed (3 of them had lung transplantation). Clinical data and their correlation with mortality are shown in Table 2 . The all-cause mortality was $45 \%$. In $25 \%$ of fatal cases S. maltophilia was regarded to have direct role in death. Twenty patients have not received specific antimicrobial therapy for S. maltophilia (13 of them died); however, patients received antibiotics against co-infective bacteria. In 11 cases colistin was applied, 9 of them were fatal. Twentynine patients were treated with SXT, 7 of them died. Six patients were treated with ciprofloxacin (5 died), 17 with moxifloxacin (3 died), 16 with levofloxacin (7 died), one with tigecycline (1 died).

The mortality was significantly associated with the following variables: ICU admission, need for mechanical ventilation, vasopressor therapy, presence of multiorgan

Table 1 Summary of MIC values and interpretations

\begin{tabular}{|c|c|c|c|c|c|c|c|}
\hline \multirow{2}{*}{$\begin{array}{l}\text { Antimicrobial agent } \\
\text { breakpoints (mg/L)* }\end{array}$} & \multirow[t]{2}{*}{ Isolates } & \multicolumn{3}{|l|}{$\mathrm{MIC}(\mathrm{mg} / \mathrm{L})$} & \multicolumn{3}{|c|}{$\%$ of isolates } \\
\hline & & MIC range & MIC 50 & MIC 90 & Resistant & Intermediate & Susceptible \\
\hline \multirow[t]{2}{*}{$\mathrm{SXT} S \leq 4, R>4$} & infective & $<0.25->32$ & 0.25 & 1 & 1 & 0 & 99 \\
\hline & colonizer & $<0.25->32$ & 0.5 & 1 & 2 & 0 & 98 \\
\hline \multirow[t]{2}{*}{ ciprofloxacin $S \leq 0.5, R>1$} & infective & $<0.5-64$ & 2 & 8 & 54 & 22 & 24 \\
\hline & colonizer & $0.5-128$ & 2 & 8 & 76 & 12 & 12 \\
\hline \multirow[t]{2}{*}{ levofloxacin $S \leq 1, R>2$} & infective & $0.125-16$ & 1 & 2 & 7 & 18 & 75 \\
\hline & colonizer & $<0.064-4$ & 0.5 & 2 & 4 & 12 & 84 \\
\hline \multirow[t]{2}{*}{ moxifloxacin $S \leq 0.5, R>1$} & infective & $<0.064-8$ & 0.25 & 1 & 7 & 6 & 87 \\
\hline & colonizer & $<0.064-4$ & 0.125 & 0.5 & 4 & 6 & 90 \\
\hline \multirow[t]{2}{*}{ doxycycline ${ }^{* *}$} & infective & $0.125-4$ & 1 & 2 & \multirow{2}{*}{\multicolumn{3}{|c|}{ Insufficient evidence }} \\
\hline & colonizer & $0.125-4$ & 1 & 2 & & & \\
\hline \multirow[t]{2}{*}{ tigecycline $S \leq 0.25, R>0.5$} & infective & $0.125-16$ & 0.5 & 2 & 50 & 38 & 12 \\
\hline & colonizer & $0.125-16$ & 0.5 & 2 & 14 & 51 & 35 \\
\hline \multirow[t]{2}{*}{ colistin $S \leq 4, R>4$} & infective & $1->256$ & 64 & $>256$ & 91 & 0 & 9 \\
\hline & colonizer & $0.25->128$ & 16 & $>128$ & 77 & 0 & 23 \\
\hline
\end{tabular}

Number of infective isolates $=77$

Number of colonizer isolates $=50$

*Breakpoints according to EUCAST. TMP-SMX breakpoints are specific for S. maltophilia. Fuoroquinolone and tigecycline breakpoints are non-species related.

Colistin breakpoints are Pseudomonas sp. specific.

**ECOFF of doxycycline of S. maltophilia is $8 \mathrm{mg} / \mathrm{l}$. 
Table 2 Univariate analysis of overall mortality of 100 patients infected by S. maltophilia

\begin{tabular}{|c|c|c|c|}
\hline & $\begin{array}{l}\text { Died } \\
(n=45)\end{array}$ & $\begin{array}{l}\text { Survived } \\
(\mathrm{n}=55)\end{array}$ & $p$ value \\
\hline & No $(\%)$ & No (\%) & \\
\hline Age in years, median (range) & $67(0-88)$ & $62(0-88)$ & - \\
\hline Gender, male & $23(51.1)$ & $35(63.6)$ & 0.29 \\
\hline Hematological malignancy & $3(6.6)$ & $3(5.4)$ & 0.31 \\
\hline Advanced cancer & $10(22.2)$ & $8(14.5)$ & 0.54 \\
\hline Diabetes mellitus & $16(35.5)$ & $16(29.1)$ & 0.22 \\
\hline Corticosteroid use & $4(8.8)$ & $8(14.5)$ & 0.31 \\
\hline Chemotherapy & $4(8.8)$ & $11(20)$ & 0.2 \\
\hline Neutropenia $(<0.5 \mathrm{G} / \mathrm{L})$ & $5(11.1)$ & $2(3.6)$ & 0.24 \\
\hline Post-transplantation stage & 0 & $5(9.1)$ & - \\
\hline Chronic heart disease & $15(33.3)$ & $18(32.7)$ & 0.88 \\
\hline Chronic kidney disease, hemodialysis & $10(22.2)$ & $5(9.1)$ & 0.12 \\
\hline Chronic lung disease & $13(28.8)$ & $14(25.4)$ & 0.87 \\
\hline Chronic liver disease & $5(11.1)$ & $7(12.7)$ & 0.95 \\
\hline Hypertension & $30(66.6)$ & $25(45.4)$ & 0.05 \\
\hline Admission to intensive care unit & $43(95.5)$ & $32(58.2)$ & 0.00005 \\
\hline Need for vasopressors & $26(57.7)$ & $8(14.5)$ & 0.00001 \\
\hline Central venous catheter & $42(93.3)$ & $29(52.7)$ & 0.00001 \\
\hline Need for mechanical ventilation & $41(91.1)$ & $28(50.9)$ & 0.00004 \\
\hline Severe sepsis, septic shock, multiorgan failure & $23(51.1)$ & $5(9.1)$ & 0.00001 \\
\hline Non-S. maltophilia bloodstream infection & $15(33.3)$ & $9(16.3)$ & 0.08 \\
\hline S. maltophilia bloodstream infection & $12(26.6)$ & $13(23.6)$ & 0.9 \\
\hline Recent surgery & $18(40)$ & $12(21.8)$ & 0.08 \\
\hline Polymicrobial infection & $35(77.7)$ & $33(60)$ & 0.09 \\
\hline
\end{tabular}

p-value $<0.05$ was considered to be significant.

Data in boldface are significant.

failure and central venous catheter. The association with mortality also remained significant after their adjustment for age and gender. Multivariate analysis with forward stepwise logistic regression identified vasopressor therapy (OR: $0.23,95 \%$ CI: 0.08-0.65, $\mathrm{p}=0.006$ ) and central venous catheter (OR: $0.15,95 \% \mathrm{CI}: 0.03-0.59, \mathrm{p}=0.007$ ) as independent determinants of mortality.

The count of WBC of infected patients ranged 0.05-37.7 Giga/L, median value was $11.2 \mathrm{Giga} / \mathrm{L}$. Values of CRP and PCT ranged 0.4-423 $\mathrm{mg} / \mathrm{L}$ and $0.15-100 \mathrm{ng} / \mathrm{mL}$, median values were $86 \mathrm{mg} / \mathrm{L}$ and $1.6 \mathrm{ng} / \mathrm{mL}$, respectively.

Other microorganisms were isolated together with $S$. maltophilia from $68 \%$ of specimens. Numbers of these isolates are shown in Table 3.

\section{Discussion}

Our study evaluated the antibiotic susceptibility of $160 \mathrm{~S}$. maltophilia isolates. In accordance with international data, SXT was found to be the most effective antimicrobial agent, so it is still the first recommended agent for infections caused by S. maltophilia. Although resistance rates are increasing, in our study only four SXT nonsusceptible isolates (2.5\%) were detected. This low resistance rate is in concordance with European and North-American data $(2-10 \%)[1,2,14,15]$.

Fluoroquinolones represent alternative treatment options of $S$. maltophilia infections. It was shown that fluoroquinolone and SXT monotherapy can achive equal efficacy [16,17]. Unlike ciprofloxacin for which nonsusceptibility was found to be high, levofloxacin and moxifloxacin were highly effective against the tested isolates in vitro. Low MIC values of moxifloxacin should be emphasized. This is in concordance with many other studies $[1,5,9,15]$. Levofloxacin and moxifloxacin have the additional advantage of disrupting S. maltophilia biofilms and reducing biofilm mass, even in subinhibitory concentrations $[1,2,18]$. Moreover, applying levofloxacin as inhalation therapy in respiratory tract infections, the achievable concentration $(50-100 \mathrm{mg} / \mathrm{L}$ ) is much higher than the highest MIC value $(16 \mathrm{mg} / \mathrm{L})$ in our study. These effects increase the clinical value of levofloxacin, especially in respiratory tract infections. It 
Table 3 Other microorganisms isolated together with 160 S. maltophilia isolates

\begin{tabular}{|c|c|c|}
\hline & $\begin{array}{l}\text { Infective } \\
(\mathrm{n}=100) \\
n\end{array}$ & $\begin{array}{l}\text { Colonizer } \\
(n=60) \\
n\end{array}$ \\
\hline \multicolumn{3}{|l|}{ Gram negative } \\
\hline Acinetobacter baumannii & 7 & 1 \\
\hline Pseudomonas aeruginosa & 24 & 7 \\
\hline other non-fermenting* & 0 & 3 \\
\hline Enterobacteriaceae ${ }^{* *}$ & 17 & 13 \\
\hline \multicolumn{3}{|l|}{ Gram positive } \\
\hline Coagulase negative Staphylococci & 4 & 9 \\
\hline Staphylococcus aureus & 8 & 7 \\
\hline Enterococcus sp. & 10 & 8 \\
\hline Candida sp. & 36 & 6 \\
\hline
\end{tabular}

*Alcaligenes faecalis, Achromobacter xylosoxidans, Pseudomonas fluorescens **Proteus mirabilis, Serratia marcescens, Escherichia coli, Klebsiella pneumoniae, Klebsiella oxytoca, Enterobacter aerogenes, Enterobacter cloacae. $\mathrm{n}$ : number of isolates.

is worth noting that the emergence of resistance to fluoroquinolones has been observed in S. maltophilia [19]. The rate of developing resistance during monotherapy was found $30 \%$ for fluoroquinolones and $20 \%$ for SXT in a previous study [16]. Hence combination therapy has been recommended in order to avoid emergence of resistance and to provide synergism between antimicrobial agents $[1,6,16,20]$. In the absence of clinical trials it is still an ongoing debate whether treatment with combination of antibiotics is superior to monotherapy. Combination therapy can be suggested in severe invasive infections and for immunocompromised patients until further clinical evidences are available [6].

Susceptibility for doxycycline was investigated instead of the often tested minocycline, since the latter is commercially not available in Hungary. Using S. maltophilia specific ECOFF, all the isolates appeared to be sensitive to doxycyline. Since ECOFF and well-established clinical breakpoints can differ significantly, it has to be assessed wether this agent is a reliable therapeutic choice. In vitro activity of tigecycline against $S$. maltophilia was determined as MIC50/MIC90 0.5/2 mg/L, which is in concordance with international data [15]. Using EUCAST non-specific breakpoints, $65 \%$ and $88 \%$ of our colonizer and infective isolates were non-susceptible to tigecycline, respectively. However, using EUCAST breakpoints established for Enterobacteriaceae $(\mathrm{S} \leq 1 \mathrm{mg} / \mathrm{L}, \mathrm{R}>$ $2 \mathrm{mg} / \mathrm{L}$ ) only $14 \%$ and $18 \%$, while with breakpoints established by USFDA for Enterobacteriaceae ( $\mathrm{S} \leq$ $2 \mathrm{mg} / \mathrm{L}, \mathrm{R}>8 \mathrm{mg} / \mathrm{L}$ ) only $10 \%$ and $4 \%$ of the isolates were non-susceptible, respectively. Due to uncertain interpretation the role of tigecycline as alternative choice in the treatment of $S$. maltophilia infections is unclear.
Clinical efficacy of tigecycline in S. maltophilia infections should be investigated. However, its synergism with SXT and colistin in combination therapy was reported $[15,21]$.

Colistin was found to have weak in vitro activity against our isolates, regardless of the testing method. Different studies showed resistance rates of S. maltophilia to be 7$100 \%$ for colistin, depending on the testing methods and the breakpoints used $[1,22]$. Our results show that colistin cannot be used in monotherapy in S. maltophilia infections; however it can show synergism with certain agents [21].

Apart from SXT, it is still not decided which testing method to use for different antimicrobial agents. EUCAST declared that antibiotic susceptibility testing of S. maltophilia is difficult since results are significantly influenced by several conditions including incubation temperature, culture medium or technique [4]. Susceptibility testing of SXT and doxycyline however was proved to be method independent and more reproducible than that of other agents [11]. Apart from these two antibiotics, interpretation is recommended after prolonged, $48 \mathrm{~h}$ incubation [11]. In our study MIC values determined after 20 hours of incubation were accepted, while CLSI method guideline was applied. Furthermore, susceptibility testing methods and breakpoints might be different depending on the site of infection: S. maltophilia isolates from the respiratory tract of patients with CF or other chronic lung diseases and isolates from bloodstream infections should be tested and interpreted in a different way [5]. Further studies are required to clarify these questions.

The majority of $S$. maltophilia strains were isolated from patients with polymicrobial infection or colonization. Interspecies interactions have an important role in bacterial virulence: S. maltophilia can protect other bacteria from antibiotics by degrading antimicrobial agents [23]. The indirect pathogenicity of $S$. maltophilia due to its $\beta$ lactamases was demonstrated [23]. While $P$. aeruginosa was the most frequent co-pathogen in our study, it should be considered that piperacillin-tazobactam, cefepime and carbapenems can be useless anti-Pseudomonas agents in an infection, where S. maltophilia expressing L1 and L2 $\beta$ lactamases is also present. Stenotrophomonas maltophilia as a co-colonizer might have a detrimental impact on $P$. aeruginosa infections treated with aminoglycosides [6]. Quorum-sensing molecules produced by S. maltophilia can also influence co-pathogen or co-colonizer bacteria $[1,24]$. Moreover, the presence of S. maltophilia in a polymicrobial community might lead to emergence of antibiotic resistance, since this species is carrying resistance plasmids or transposons that facilitate the spread of resistance integrons to other bacterial species [1]. Species belonging to Enterobacteriaceae family were the second most frequent co-pathogens in this study, including even 
multidrug resistant, VIM-4 carbapenemase producing Enterobacter cloacae strains [25]. Whether S. maltophilia affected the resistance pattern of $E$. cloacae strains or vica $v e r s a$, is a further complex question. It is yet to be clarified whether drug resistance determinants are transmitted between S. maltophilia and other bacteria within biofilms [5]. Other microorganisms causing infection together with $S$. maltophilia can influence the clinical outcome as well [16].

Based on partial gyrB gene sequences, S. maltophilia complex can be divided into distinct groups which differ in antimicrobial resistance rates [26]. Further investigations are required to clarify whether there is a relation between the protein mass spectra of $S$. maltophilia group and antibiotic resistance.

Most infections caused by S. maltophilia were associated with severe morbidity and long-term, extensive ICU treatment. Previously published mortality rates vary between 14-62\% [16]. The high all-cause mortality rate (45\%) we observed can be connected to the serious underlying illnesses rather to $S$. maltophilia itself. However, the strict attributable mortality rate (11\%) was also high, therefore clinical significance of S. maltophilia infections must be considered particularly in patients admitted to ICUs [27]. The fact that nearly $70 \%$ of infective isolates were collected from lower respiratory tract samples has to be considered as a limitation of our study.

\section{Conclusions}

S. maltophilia is still a challenging multiresistant nosocomial pathogen. Our results show that SXT is the most potent antibiotic drug against S. maltophilia. Due to the low frequency of moxifloxacin and levofloxacin resistance, these agents can be used either in high dose monotherapy or rather in combination with other antibiotics, concerning the chance of rapid resistance development during monotherapy. The establishment of clinical breakpoints for agents other than SXT is strongly required in the near future. The most reliable antibiotic susceptibility testing method for alternative antibiotics should urgently be declared. Clinicians have to consider that $S$. maltophilia as a co-pathogen or co-colonizer in polymicrobial infections can have negative impact on the success rate of antibiotic treatment and clinical outcome.

\section{Competing interests}

The authors declare that they have no competing interests.

\section{Authors' contributions}

JE, KK conceived and designed the experiments. JE, KG, GG performed the experiments. JE, KK, PJ analysed the data. LGy, KK contributed reagents/ materials. JE wrote the manuscript. JE, KG, PJ, KK, LGy corrected the manuscript. All authors read and approved the final manuscript.

\section{Author details}

'Diagnostic Laboratory of Clinical Microbiology, Institute of Laboratory Medicine, Semmelweis University, Budapest, Hungary. ${ }^{2}$ Institute of Medical
Microbiology, Semmelweis University, Budapest, Hungary. ${ }^{3}$ Hungarian Defence Forces, Military Medical Centre, Budapest, Hungary.

Received: 19 August 2014 Accepted: 9 December 2014

Published online: 31 December 2014

\section{References}

1. Abbott IJ, Slavin MA, Turnidge JD, Thursky KA, Worth LJ: Stenotrophomonas maltophilia: emerging disease patterns and challenges for treatment. Expert Rev Anti Infect Ther 2011, 9(4):471-488.

2. Brooke JS: Stenotrophomonas maltophilia: an emerging global opportunistic pathogen. Clin Microbiol Rev 2012, 25(1):2-41.

3. Falagas ME, Kastoris AC, Vouloumanou EK, Dimopoulos G: Communityacquired Stenotrophomonas maltophilia infections: a systematic review. Eur J Clin Microbiol Infect Dis 2009, 28(7):719-730.

4. http://www.eucast.org/antimicrobial_susceptibility_testing/guidance_documents. Stenotrophomonas maltophilia EUCAST guidance note 20120201.

5. Brooke JS: New strategies against Stenotrophomonas maltophilia: a serious worldwide intrinsically drug-resistant opportunistic pathogen. Expert Rev Anti Infect Ther 2014, 12(1):1-4.

6. Looney WJ, Narita M, Mühlemann K: Stenotrophomonas maltophilia: an emerging opportunist human pathogen. Lancet Infect Dis 2009, 9(5):312-323.

7. Ford BA, Burnham CA: Optimization of routine identification of clinically relevant Gram-negative bacteria by use of matrix-assisted laser desorption ionization-time of flight mass spectrometry and the Bruker Biotyper. J Clin Microbiol 2013, 51(5):1412-1420.

8. Anzai Y, Kim H, Park JY, Wakabayashi H, Oyaizu H: Phylogenetic affiliation of the pseudomonads based on 16S rRNA sequence. Int J Syst Evol Microbiol 2000 Jul; 50(Pt 4):1563-1589.

9. CLSI: Performance Standards for Antimicrobial Susceptibility Testing: Twentieth Informational Supplement, M100-S20. Wayne, PA: Clinical and Laboratory Standards Institute; 2010.

10. Garcia LS: Clinical Microbiology Procedures Handbook. Washington: ASM press; 2010.

11. Carroll KC, Cohen S, Nelson R, Campbell DM, Claridge JD, Garrison MW, Kramp J, Malone C, Hoffmann M, Anderson DE: Comparison of various in vitro susceptibility methods for testing Stenotrophomonas maltophilia. Diagn Microbiol Infect Dis 1998, 32(3):229-235.

12. EUCAST clinical breakpoints: http://www.eucast.org/clinical_breakpoints/.

13. Silbert S, Pfaller MA, Hollis RJ, Barth AL, Sader HS: Evaluation of three molecular typing techniques for nonfermentative Gram-negative bacilli. Infect Control Hosp Epidemiol 2004, 25(10):847-851.

14. Toleman MA, Bennett PM, Bennett DM, Jones RN, Walsh TR: Global emergence of trimethoprim/sulfamethoxazole resistance in Stenotrophomonas maltophilia mediated by acquisition of sul genes. Emerg Infect Dis 2007, 13(4):559-565.

15. Farrell DJ, Sader HS, Jones RN: Antimicrobial susceptibilities of a worldwide collection of Stenotrophomonas maltophilia isolates tested against tigecycline and agents commonly used for S. maltophilia infections. Antimicrob Agents Chemother 2010, 54(6):2735-2737.

16. Wang YL, Scipione MR, Dubrovskaya Y, Papadopoulos J: Monotherapy with fluoroquinolone or trimethoprim-sulfamethoxazole for treatment of Stenotrophomonas maltophilia infections. Antimicrob Agents Chemother 2014, 58(1):176-182.

17. Cho SY, Kang Cl, Kim J, Ha YE, Chung DR, Lee NY, Peck KR, Song JH: Can levofloxacin be a useful alternative to trimethoprimsulfamethoxazole for treating Stenotrophomonas maltophilia bacteremia? Antimicrob Agents Chemother 2014, 58(1):581-583.

18. Wu K, Yau YC, Matukas L, Waters V: Biofilm compared to conventional antimicrobial susceptibility of Stenotrophomonas maltophilia Isolates from cystic fibrosis patients. Antimicrob Agents Chemother 2013, 57(3):1546-1548.

19. Wu H, Wang JT, Shiau YR, Wang HY, Lauderdale TL, Chang SC, TSAR Hospitals: A multicenter surveillance of antimicrobial resistance on Stenotrophomonas maltophilia in Taiwan. J Microbiol Immunol Infect 2012 , 45(2):120-126.

20. Falagas ME, Valkimadi PE, Huang YT, Matthaiou DK, Hsueh PR: Therapeutic options for stenotrophomonas maltophilia infections beyond co-trimoxazole: a systematic review. J Antimicrob Chemother 2008, 62(5):889-894. 
21. Betts JW, Phee LM, Woodford N, Wareham DW: Activity of colistin in combination with tigecycline or rifampicin against multidrug-resistant Stenotrophomonas maltophilia. Eur J Clin Microbiol Infect Dis 2014, [Epub ahead of print].

22. Biswas S, Dubus JC, Reynaud-Gaubert M, Stremler N, Rolain JM: Evaluation of colistin susceptibility in multidrug-resistant clinical isolates from cystic fibrosis, France. Eur J Clin Microbiol Infect Dis 2013, 32(11):1461-1464.

23. Kataoka D, Fujiwara H, Kawakami T, Tanaka Y, Tanimoto A, Ikawa S, Tanaka Y: The indirect pathogenicity of Stenotrophomonas maltophilia. Int J Antimicrob Agents 2003, 22(6):601-606

24. Sherrard $L$, Tunney MM, Elborn JS: Antimicrobial resistance in the respiratory microbiota of people with cystic fibrosis. Lancet 2014, 384(9944):703-713.

25. Juhász E, Jánvári L, Tóth A, Damjanova I, Nobilis A, Kristóf K: Emergence of VIM-4- and SHV-12-producing Enterobacter cloacae in a neonatal intensive care unit. Int J Med Microbiol 2012, 302(6):257-260.

26. Rhee JY, Choi JY, Choi M, Song JH, Peck KR, Ko KS: Distinct groups and antimicrobial resistance of clinical Stenotrophomonas maltophilia complex isolates from Korea. J Med Microbiol 2013, 62(Pt 5):748-753.

27. Samonis G, Karageorgopoulos DE, Maraki S, Levis P, Dimopoulou D, Spernovasilis NA, Kofteridis DP, Falagas ME: Stenotrophomonas maltophilia infections in a general hospital: patient characteristics, antimicrobial susceptibility, and treatment outcome. PLoS One 2012, 7(5):e37375.

\section{Submit your next manuscript to BioMed Central and take full advantage of:}

- Convenient online submission

- Thorough peer review

- No space constraints or color figure charges

- Immediate publication on acceptance

- Inclusion in PubMed, CAS, Scopus and Google Scholar

- Research which is freely available for redistribution 